\title{
Radial Poiseuille Flow of a Homeotropic Nematic LC Layer
}

\author{
Karl Hiltrop and Fred Fischer \\ Physikalisches Institut der Westfälischen Wilhelm-Universität, Münster
}

(Z. Naturforsch. 31 a, 800-807 [1976] ; received May 10, 1976)

\begin{abstract}
A two-dimensional Poiseuille flow in a radial direction allows to study simultaneously a whole range of flow rates. Three different deformation modes of the director field have been observed, the 'bow' mode, the 'screw' mode, and the 'peak' mode. Above a critical flow rate the bow mode transforms into the screw mode. Right and left screw mode can be produced side by side. The peak mode reached by temporary high flow rates is metastable at low flow rates. Quantitative results are reported and discussed.

By reversing the flow direction in the screw mode region the left screw mode is transformed into the right screw mode and vice versa.

By reversing the flow direction in the peak mode region special tube-like deformation structures are obtained. Each open tube has a point disclination at either end. Both are of opposite strength \pm 1 .
\end{abstract}

\section{§ 1. Introduction}

Many instabilities and deformation modes, which can be produced by shear flow, have been discovered by Pieranski and Guyon ${ }^{1}$. They have experimented with Couette flow as well as with Poiseuille flow (also named Hagen-Poiseuille flow), using homogeneous boundary conditions and rectangular LC cells in which the flow velocity profile is constant over the cell.

We have studied the shear flow of a homeotropic layer of MBBA using a new radial flow geometry in a two-dimensional Hagen-Poiseuille flow cell. Our main interest has been directed towards the analysis of different new deformation modes of the director field.

The flow tries to deform the initial homeotropic orientation of the layer in a way that the director is tilted towards an orientation of lower viscosity. The viscous torque is balanced by the elastic one. In the middle plane between the glass walls the director stays in its initial orientation because of symmetry reasons. Altogether a 'bow' like deformation of the director field lines may be expected.

However with increasing flow rate the elastic splay-bend energy of the bow is also increased. A transition into another deformation mode is expected, in which the bow is broken in the middle of the layer. The director field lines now form a 'peak'. First unexpected for us, a third deformation mode occurs which we have called 'screw' mode.

The response of the different deformation modes against flow reversal with and without external fields

Reprint requests to Physikalisches Institut der Universität, Schloßplatz 7, D-4400 Münster. has also been studied. In $\S 2$ experimental details and in $\S 3$ experimental results are presented. In $\$ 4$ quantitative evaluations of the results are discussed.

\section{§ 2. Experimental Details}

A sketch of the flow cell used in our experiments is shown in Figure 1. The LC layer - we use MBBA throughout these investigations - is enclosed be-

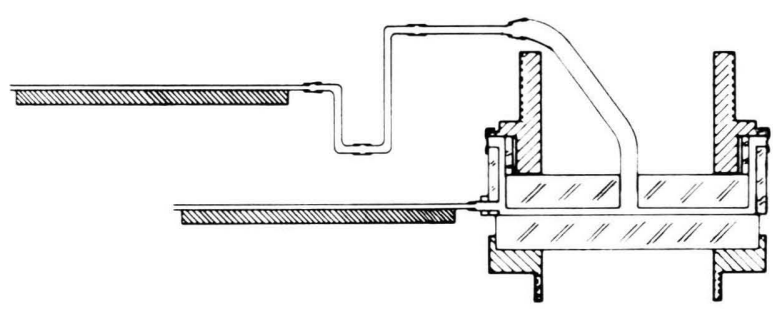

Fig. 1. Sketch of the radial flow zell. The pressure difference is produced by a vertical shift of the upper horizontal tube. (Its $\mathrm{U}$ shaped connection stands out of the drawing plane.)

tween two circular plane glass plates with adjustable distance $d$. Through a central hole in the upper plate the LC can flow in and out. A short glass tube glued to the lower plate forms the outer wall of the LC reservoir surrounding the LC layer. The radial flow arrangement allows the simultaneous observation of a whole range of flow rates. The flow rate decreases inversely proportional to the radius $r$. A constant pressure difference $\Delta p$ between the central $\left(r_{1}=2.5 \mathrm{~mm}\right)$ and the outer $\left(r_{2}=35 \mathrm{~mm}\right)$ end of the radial flow cell is supplied by two horicontally adjusted glass tubes of the same inner diameter of about $4 \mathrm{~mm}$. They are connected by silicone hoses to the central hole and to the outer LC reservoir respectively. Before filling each glass tube has to be 
cleaned carefully in order to become uniformly wetted by the LC meniscus. Otherwise an uncon. trollable pressure difference could arise from unequal shapes of the LC surfaces. By vertically shifting the glass tube connected to the upper plate the pressure difference $\Delta p$ can be steadily varied between plus and minus $200 \mathrm{~mm}$ MBBA column with an accuracy of $\pm 10^{-2} \mathrm{~mm}$. This yields a very simple and inexpensive equipment for producing and measuring small and constant pressure differences.

A molecular layer of lecithin establishes strong homeotropic anchoring at the glass walls. The equipment for observing and measuring the average optical anisotropy of the LC film in a telecentric beam of monochromatic light $(546 \mathrm{~nm})$ between crossed polarizers is the same as used before ${ }^{2}$. Simultaneously the LC film can locally be observed with a conoscopic beam of light when needed.

For the sake of clearness we define a local Carthesian coordinate system with its $z$ axis normal to the layer. At every place of the layer the positive $x$ axis is directed radially outwards.

\section{§ 3. Experimental Results}

In our experiments we have found three different deformation modes of the director field, stable or metastable, within certain limits of flow rates or depending on the history of pretreatment. We call them 'bow' mode, 'screw' mode, and 'peak' mode on account of the shape of their director field lines.

\section{a) The Bow Mode}

At low flow rates one expects a nearly parabolic profile of the flow velocity $v_{x}(z)$ (Figure 2 ). The viscous torque upon the director is largest at the walls $(z= \pm d / 2)$ and zero in the middle of layer $(z=0)$. Therefore near the walls the director attains its maximum alignment angle $\vartheta_{\mathrm{m}}$ relative to the initially homeotropic orientation. Towards the middle of the layer the alignment angle $\vartheta$ decreases and
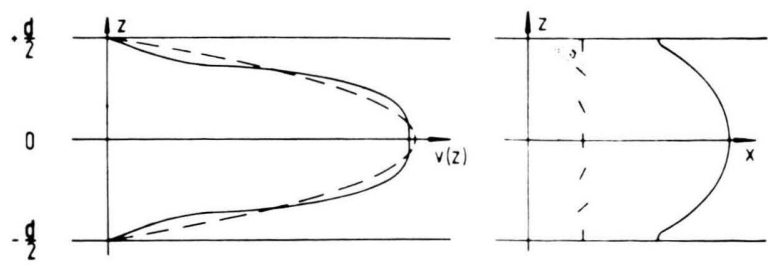

Fig. 2. Velocity profile (dashed curve for isotropic liquid) and director field in different presentations. reverses sign at $z=0$. The director field lines have the typical shape of a bow (Figure 2).

The flow alignment angle averaged over the thickness $d$ determines the local average optical anisotropy of the layer. Hence the telecentric interference pattern consists of concentric dark circles. Figure 3
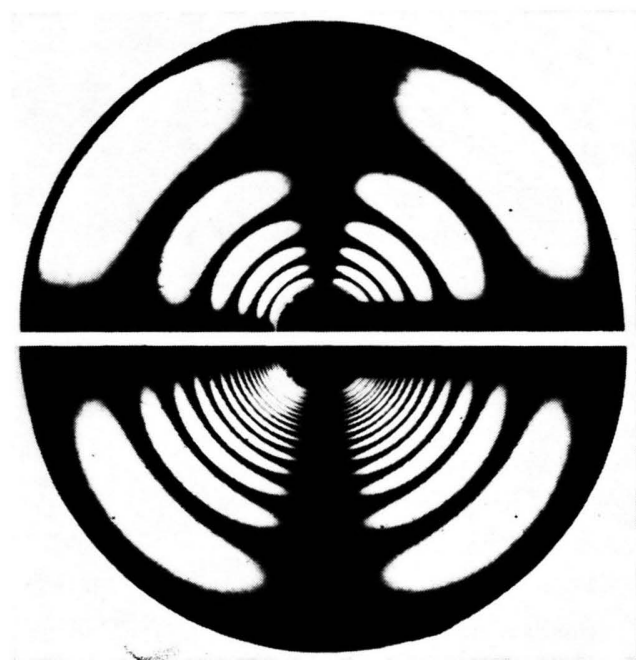

Fig. 3. Interference pattern of bow mode. The upper part belongs to a thickness $d=45 \mu \mathrm{m}$ and $\Delta p=10 \mathrm{~mm}$ MBBA column. The lower part belongs to $d=200 \mu \mathrm{m}$ and $\Delta p=$ $0.1 \mathrm{~mm}$ MBBA column.

gives two examples for different layer thicknesses. The interference order $m$ decreases with increasing $r$. The order number $m=0$ is seen at the outer circumference. The dark cross indicates the orientation of the two polarizers.

\section{b) The Screw Mode}

By increasing the pressure differences above a certain limit $\Delta p_{\mathrm{c}}$ which depends on the thickness $d$ a new pattern gushes out of the central part of the flow cell. It needs some minutes until the bow mode starts to transform into this new 'screw' mode. Within an area of certain radius $r_{0}$ a new stationary state is reached, as shown in Fig. 4, during a time which increases with layer thickness $d$. In very clean MBBA $\left(T_{\mathrm{c}} \geqq 46{ }^{\circ} \mathrm{C}\right)$ many closed lines remain near the border line to the bow mode area (Figure 4 a). They indicate the presence of two kinds of screw modes, namely left and right screw modes. They are separated by walls with a pure bow mode deformation. Only within hours one kind of domains may disappear. In less clean MBBA this domain 


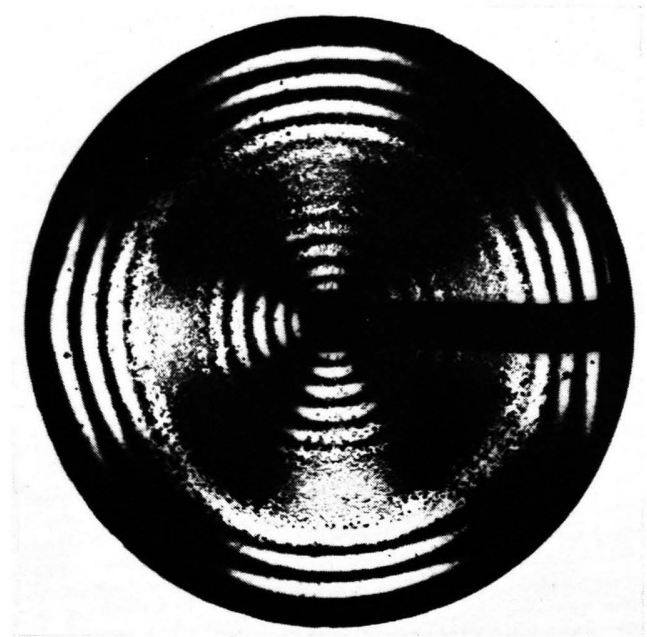

Fig. 4 a. Left and right screw mode domains of pure MBBA near the transition to the bow mode (outer part).

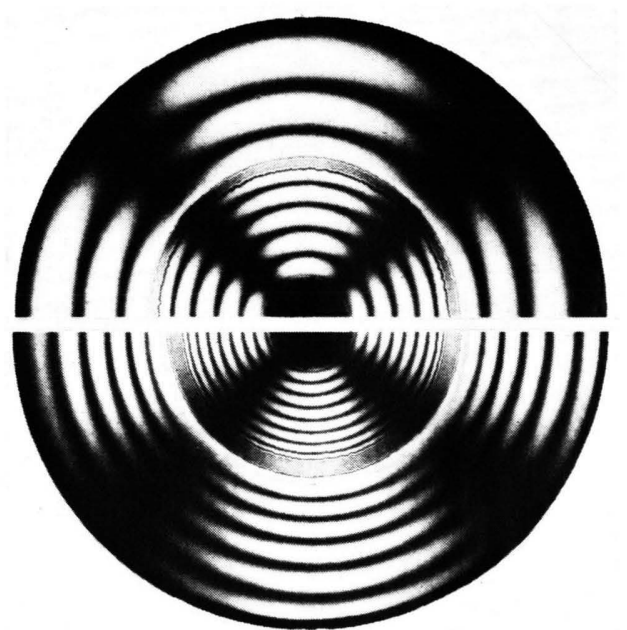

Fig. 4 b. Screw mode region with only one screw sence (impure MBBA) within the bow mode region. Upper part $d=$ $50 \mu \mathrm{m}$, lower part $d=100 \mu \mathrm{m}$.

pattern disappears within minutes and one of the two screw modes dominates (Figure $4 \mathrm{~b}$ ). A weak preference of the left or right helix has been observed. We suspect traces of various optical active impurities to cause this effect.

One observes a dense ring system near the border line to the bow mode area. In Fig. 4 a as well as in Fig. $4 \mathrm{~b}$ we observe around the transition radius $r_{0}$ a gray diffuse ring which destroys every interference also within the dark cross.

After stopping the flow the LC layer returns to the homeotropic orientation within very different times. While the bow mode relaxes within seconds the screw mode eventually needs some minutes. During that process the dark rings fade and the bright rings grow into the dark cross.

Byturning one of the polarizers by a certain angle $\alpha$ the black cross is regained but now rotated by an angle $\alpha / 2$. During the time of relaxation the angle $\alpha$ steadily increases from 0 to $\pi$.

These facts shall be explained as follows: With increasing flow rate more and more splay-bend energy is stored in the middle part of the layer. It has been shown for the case of pure splay-bend without flow that by exceeding a critical deformation the bow mode becomes unstable and the director breaks out into the third dimension ${ }^{3}$. The same happens here with flow which only serves to accomplish the splay-bend deformation. In Fig. $5 \mathrm{a}$ and $\mathrm{b}$ this bowscrew transition is represented.

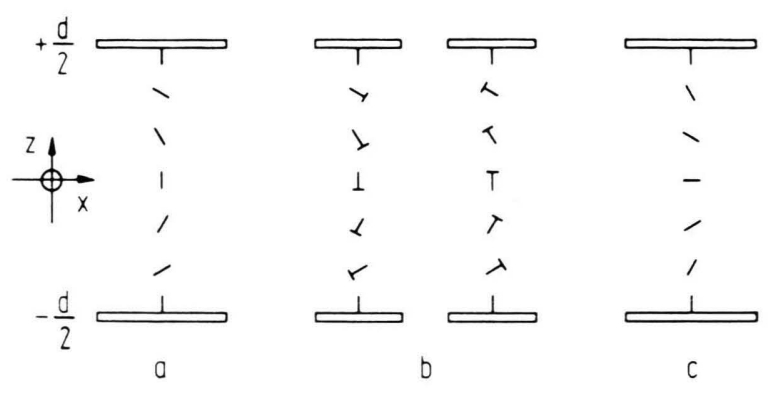

Fig. 5. Director orientation in the layer: a) bow mode: b) right and left screw mode; c) peak mode.

In the screw mode the projection of the director into the layer plane performs a full $\pi$ turn when moving from $z=-d / 2$ to $z=+d / 2$. As soon as the Mauguin condition ${ }^{4}$ is fulfilled the electric vector of linear polarized light travelling normal through the layer also performs a full $\pi$ turn. Therefore one can understand why also in the screw mode the dark cross appears without distortion.

The bow-screw transition is a kind of second order transition. In the screw mode left and right turn are strictly equivalent in case of a pure nematic LC.

The gray ring at the border of the bow mode region to the screw mode region is the result of a critical opalescence caused by large scale fluctuations of the director field. Hereby the linear polarization of the light beam gets lost. It might be worthwhile to study this phenomenon more closely.

The different relaxation times of the bow mode and of the screw mode after stopping the flow can 
be understood qualitatively from their different deformation of the director field. In the bow mode the main deformation is concentrated near the walls. So the relaxation is as fast as in a much thinner LC layer after simple shear flow. In the screw mode we have the additional screw deformation which spans over the whole thickness $d$ of the sample. Therefore the relaxation is slower.

The existence of the screw mode has been confirmed by conoscopic observations. The conoscopic technique allows to observe the inclination of the director out of the bow plane and to distinguish between left and right screws.

What happens in the screw mode when the flow direction is suddenly reversed? The left screw mode is transformed into the right screw mode and vice versa. This has been proved with the conoscopic technique. Apparently the inclination of the director in the middle of the layer cannot be changed while the flow alignment near the glass walls is turned over. A consequence of this behaviour leads to the following experiment. After we have produced a screw mode area with screw sense favourable for that sample, the flow direction is suddenly reversed and simultaneously increased. So the new stationary screw mode should cover the full Poiseuille cell. Figure 6 shows the result. After 1 min (Fig. 6 a, upper part) the curled domain pattern develops within the bow mode area. After 2 min it covers the bow mode area completely. After 3 min the interference field clears up again (Fig. 6 a, lower part). We observe a boundary wall between the initial screw mode area, now slightly reduced in size, and the new outer screw mode area. While the new one has the more favourable screw sense, the old one has been transformed into the less favourable screw sense.

The wall between both domains contains elastic energy and tries to shrink in length. However, when the LC flows outwards the less favourable domain may increase. By adjusting the flow rate the wall will be stabilized at any radius $r_{0}$ you want, as long as the LC layer stays in the screw mode. Experiments of this kind could be used to compare the "line force' with the 'flow resistance force' of the wall.

The wall between left and right screw mode domain becomes better visible after the flow has been stopped. Figure $6 \mathrm{~b}$ shows such a circular wall as a dark line. While the wall with its internal bow mode deformation has already relaxed into the

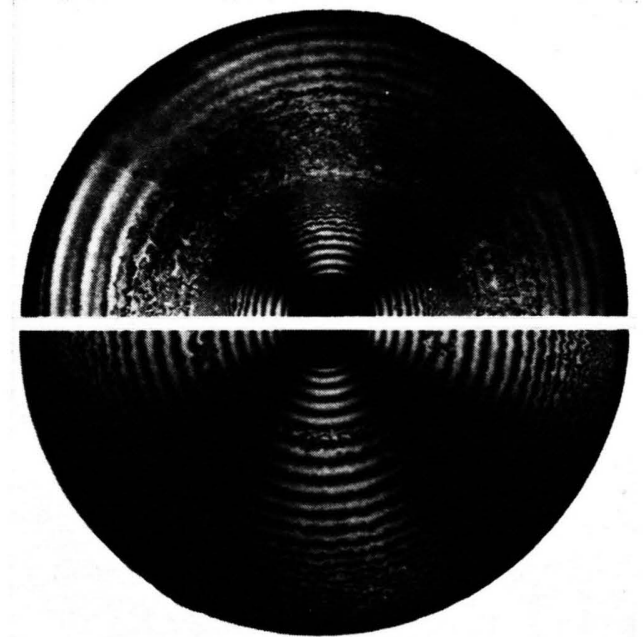

Fig. 6 a. Development of a circular wall between left and right screw domain by suddenly reversing and increasing the flow. Upper part after $1 \mathrm{~min}$, lower part after $3 \mathrm{~min}$.

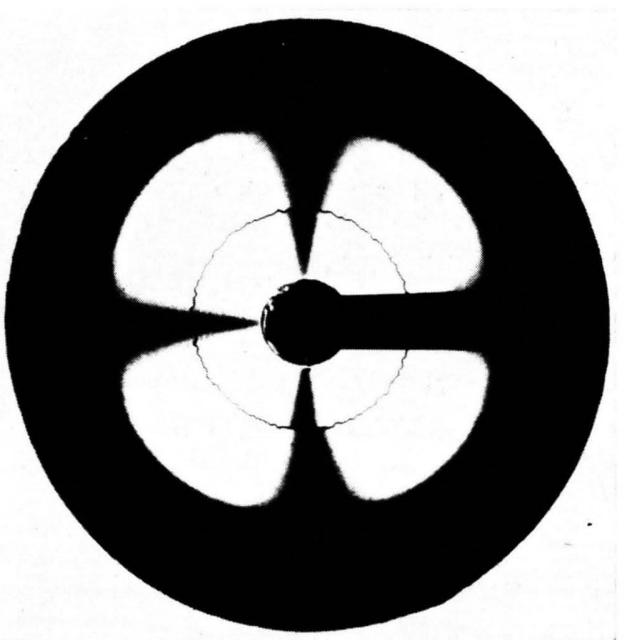

Fig. $6 \mathrm{~b}$. The wall, after stopping the flow, already relaxed into the homeotropic orientation because of its bow mode structure.

homeotropic orientation, the left and right screw mode domains still show some residue of birefringence.

The existence of right and left screw modes suggests to control the screw sense by external fields, for instance by magnetic fields. Their torque density is $\boldsymbol{\Gamma}=\mu_{0} \chi_{\mathrm{a}}(\boldsymbol{n} \boldsymbol{H})(\boldsymbol{n} \times \boldsymbol{H})$. With a positive $\chi_{\mathrm{a}}=$ $\chi \|-\chi_{\perp}$ the director might be turned in a way to become parallel to the local magnetic field direction. So in a weak magnetic field with oblique orientation 
to the Poiseuille cell the homeotropic orientation becomes slightly deformed already without any flow.

When we now add the Poiseuille flow the decision for left or right screw mode is already done in the bow mode region. Then the growing screw mode area is devided into two halves, separated by a straight wall (Figure 7 a). The magnetic field can be removed now. From its orientation we know what part is the left screw mode and what is the right. Inside the straight wall the original bow mode deformation causes a reduction of the optical anisotropy. Very impressive are the broad orifices of the wall into the bow mode area.

In less clean samples of MBBA the high symmetry of Fig. 7 a could not be obtained. If the oblique magnetic field is not too strong the two screw mode domains develop into different sizes (Figure $7 \mathrm{~b})$. After the magnetic field has been removed,

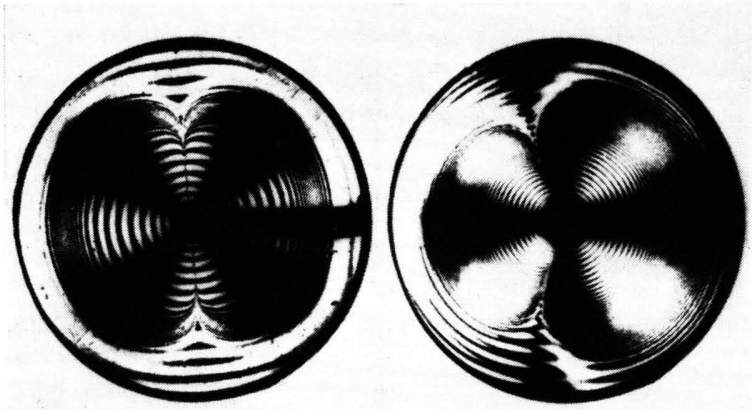

Fig. 7. Left and right screw domain separated by a wall initiated by oblique magnetic field. a) Equal size in pure MBBA, b) unequal size in impure MBBA.

while the flow is continuing, the bigger domain grows at the expense of the smaller one until the less favourable domain has disappeared. But by applying the oblique magnetic field again the other screw domain reappears on the same side.

\section{c) The Peak Mode}

By further increasing the flow rate another deformation mode occurs. If the LC flows inwards one observes tongue-shaped regions developing at the periphery and floating inwards too. Figure 8 gives and example. Near the tip of each tonque the interference order of both bow and screw modes becomes increased. This shows that the flow resistance in the tongues is lower than in the bow or screw mode region. By an outwards flow the tongues are produced
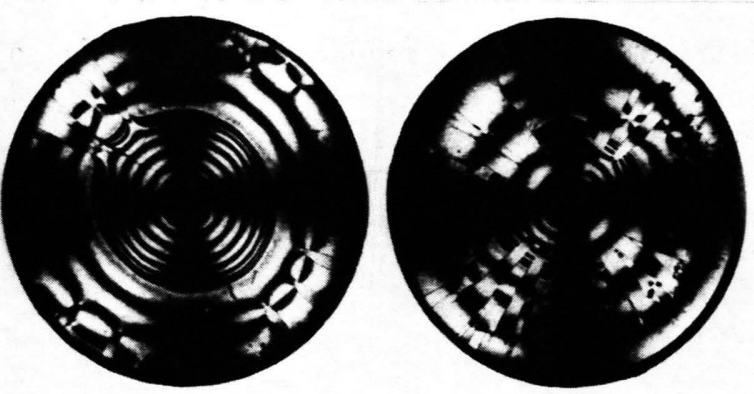

Fig. 8. Growing of tonque shaped peak mode area from the circumference to the center. a) After about $10 \mathrm{~min}$, b) after about $40 \mathrm{~min}$.

at the edge of the central hole. A temporary very high flow rate transforms the whole layer.

This new deformation mode also shows interference rings. After switching off the flow all these rings disappear within a few seconds without returning to the homeotropic state. There remains a certain constant birefringence. The return to the homeotropic orientation takes place over a nucleation process. Often the layer becomes covered with many homeotropic spots, which grow slowly and join with neighbouring spots.

From these observations as well as from shear flow experiments by Waltermann and Fischer ${ }^{5}$ we conclude that in the new mode the director makes a $\pi$ turn between $z=-d / 2$ and $z=+d / 2$, as shown in Figure 5 c. From the peaked shape of the director field lines we call it 'peak' mode. It is suitable to define a unit vector $\mathbf{c}$ describing the orientation of the peak in the $x-y$ plane. We call it $\mathrm{C}$ rector. A peak region is separated from a bow or screw region by a disclination of the strength $\pm \frac{1}{2}$. A system of interference lines due to the deformation field around the disclination can be observed under the microscope.

The interference order of the peak mode at rest has been obtained by Waltermann ${ }^{6}$ using a numerical calculation. Experimentally this order is determined utilizing the fact that at high flow rates the direktor attains the flow alignment in almost the whole layer, independently of the deformation mode. So the interference pattern of neighbouring peak and screw mode regions will coincide in the interference order. At these high flow rates the screw mode is not stable. Therefore one has to count quickly. The results is $m / d=(19.3 \pm 0.6) \times 10^{4} \mathrm{~m}^{-1}$ compared with $\mathrm{m} / d=19.7 \times 10^{4} \mathrm{~m}^{-1}$ calculated by Waltermann, using $\Delta n=0.233$ and $K_{1} / K_{3}=0.82$. 
The $\mathrm{C}$ vector tries to orientate parallel to the flow direction. A reversal of the flow direction results in a reversal of the $\mathrm{C}$ vector. However, the $\pm \pi$ turn around the $z$ axis does not start immediately after reversing the flow. At low flow rates the $\mathrm{C}$ vector stays opposite to the flow direction. The order of interference diminishes, as can also be observed at the conoscopic pattern. It has not yet been decided clearly enough if this is a metastable flow situation with hysteresis effects or if only the $\pm \tau$ turn develops very slowly.

Without external influence $+\pi$ turn and $-\pi$ turn of the $\mathrm{C}$ vector take place simultaneously in different regions of the layer. The $+\pi$ and.$- \pi$ regions are separated by walls in which the $\mathrm{C}$ vector has not been turned at all. By crossing such a wall from $-\pi$ to $+\pi$ region the $\mathrm{C}$ vector makes a full $2 \pi$ turn. Obviously $+\pi$ and $-\pi$ turned regions are identical and cannot be distinguished.

So what we first have named walls are in reality more a kind of deformation tubes. Such a deformation tube is very stable even at high flow rates. It may rupture and produce pairs of point disclinations with opposite strength \pm 1 . Figure 9 a gives an example after the flow has been stopped. In a homo. geneous field of the $\mathrm{C}$ vector the deformation tube appears in form of four parallel black stripes on a white background, which can be realized by a

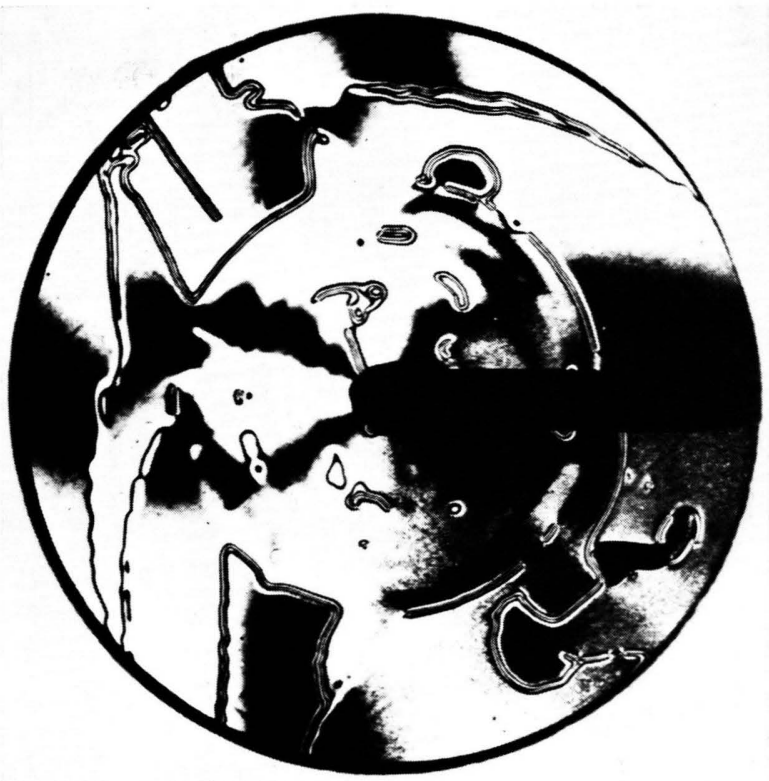

Fig. 9 a. Deformation tubes in the peak mode produced by reversing the flow, observed in the flow-less state.

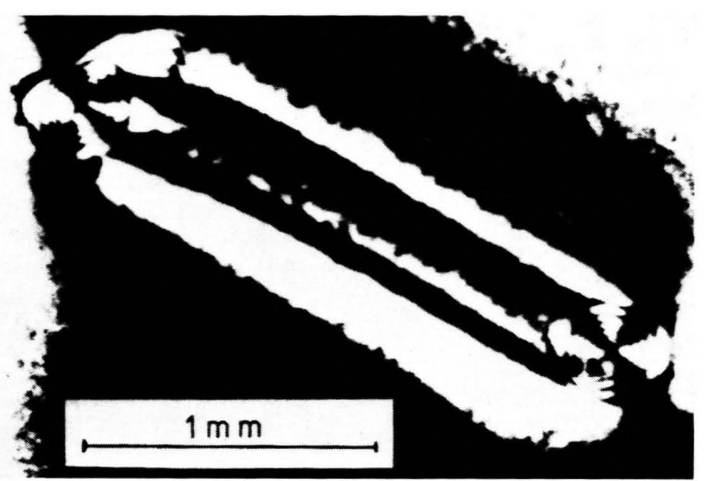

Fig. 9 b. Short deformation tube with point disclinations at its ends.

suitable rotation of the crossed polarizers. Figure $9 \mathrm{~b}$ shows a magnified fragment of a deformation tube with point disclination of opposite strength at both ends.

Therefore the deformation tubes have a polar structure. It can be recognized by a simultaneous rotation of the crossed polarizers. While in the +1 point disclination the dark cross rotates in the same sense it rotates in the opposite sense in the -1 point disclination.

In the following we describe the additional influence of a constant magnetic field parallel to the layer. It causes a torque upon the $\mathrm{C}$ vector which tries to orientate it parallel or antiparallel to this field direction (for $\chi_{\mathrm{il}}>0$ ). Let us assume that the crossed polarizers make an angle of $45^{\circ}$ with the horizontal magnetic field. At the beginning the $\mathrm{C}$ vector has been directed radially to the center of the cell. With increasing time the beams of the black cross indicating the places where the $\mathrm{C}$ vector is parallel or normal to the vector of the incoming light, are turned in opposite sense until they coincide. Simultaneously the conoscopic picture, cross faded into the telecentric picture, shows the local rotation of the $\mathrm{C}$ vector directly by a rotation of the interference pattern.

When now the flow is switched on in a direction opposite to the initial direction of the $\mathrm{C}$ vector the flow will continue to turn the $\mathrm{C}$ vector in the same sense as the magnetic field has already done. With a high enough flow rate the $C$ vector will have performed more than a full $\pm \pi$ turn. Hereby hysteresis phenomena may be observed. There are four quadrants in which the rotation takes place in alternately opposite sense. They are separated by deformation 
tubes. After switching off the flow the magnetic field reorients the $\mathrm{C}$ vector in the same way as described above so that the rotation of the $\mathrm{C}$ vector is continued in the same sense.

Now the flow can be switched on again in the reversed direction. This produces a further rotation of the $\mathrm{C}$ vector. By that way the boundary between two quadrants becomes a deformation tube in which the $\mathrm{C}$ vector endures a $4 \pi$ turn. It has to be checked in further experiments how many such turns of the $\mathrm{C}$ vector between the quadrants can be produced by continuing this procedure.

\section{§ 4. Quantitative Evaluation and Discussion}

The pure flow deformation without external fields has been measured more quantitatively. About 60 photographed interference patterns with certain pressure differences $\Delta p$ between 0.1 and $30 \mathrm{~mm}$ MBBA column $( \pm 2 \%)$ and layer thickness $d$ between $60 \mu \mathrm{m}$ and $120 \mu \mathrm{m}( \pm 2 \%)$ have been analysed. That means for each interference order $m$ the radius $r$ of the interference ring had to be determined.

A scale analysis shows that

$$
m / d=f\left(\frac{1}{\langle\eta\rangle} \frac{\Delta p d^{3}}{r}\right)
$$

where $\langle\eta\rangle$ is a mean viscosity averaged over the thickness and over the length $\left(r_{2}-r_{1}\right)$ of the flow cell. $\langle\eta\rangle$ itself comes out to be also a function of $\Delta p \mathrm{~d}^{3}$. First $m / d$ has been plotted as a function of $\Delta p d^{3} / r$. Each photograph gave its own curve. By changing all abszissa data of each curve by a suitable chosen constant factor $A$ all curves of the bow and screw mode as well as all curves of the peak mode could be adapted to one another. This factor is proportional to $\langle\eta\rangle^{-1}$. The proportionality factor is unknown and different for both curves. Figure 10 shows the result. The lower curve starts parabolically at the origin and describes the bow mode behaviour. At about $m / d=1.1 \times 10^{5} \mathrm{~m}^{-1}$ we have the transition into the screw mode. We observe a fast rise of $m / d$ corresponding to the high ring density in the pictures. Above $m / d \approx 2.7 \times 10^{5} \mathrm{~m}^{-1}$ we obtain a rather sharp bend over into the saturation range. The limiting value of complete shear flow alignment is $(m / d)_{\max }=4.17 \times 10^{5} \mathrm{~m}^{-1}$ for MBBA at room temperature ${ }^{2}$. This limit is approached very slowly.

The upper curve belongs to the peak mode. $m / d$ in the peak mode is always larger than in the bow

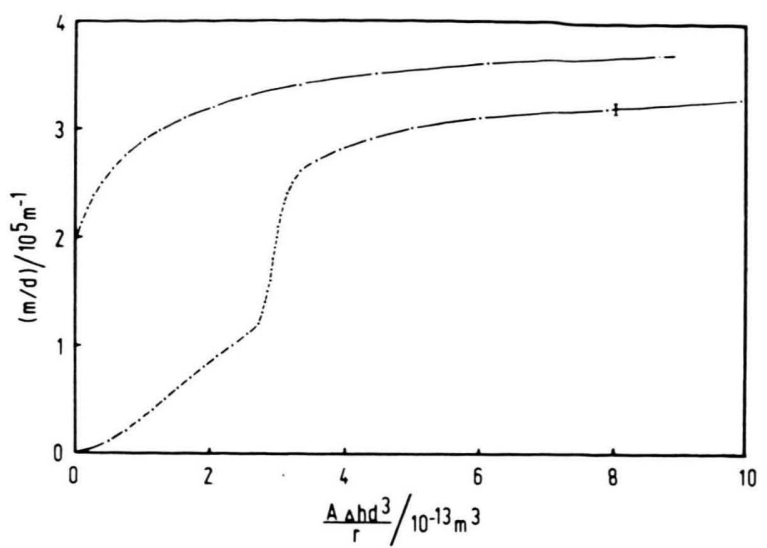

Fig. 10. Effective optical anisotropy $(m / d)$ versus $A \Delta h d^{3} / r$ which is proportional to the flow rate $\left[A=f\left(\Delta h d^{3}\right) ; \Delta h=\right.$ $\Delta p /$ ombBA]. Upper curve: peak mode. Lower curve: bow and screw mode.

or screw mode. At zero flow it has a value $m / d=$ $1.9 \times 10^{5} \mathrm{~m}^{-1}$. The curve starts with a finite slope. As experiments have shown, this curve can be extended to negative abszissa values; however, if stable or not has not yet been certified. Also the bow

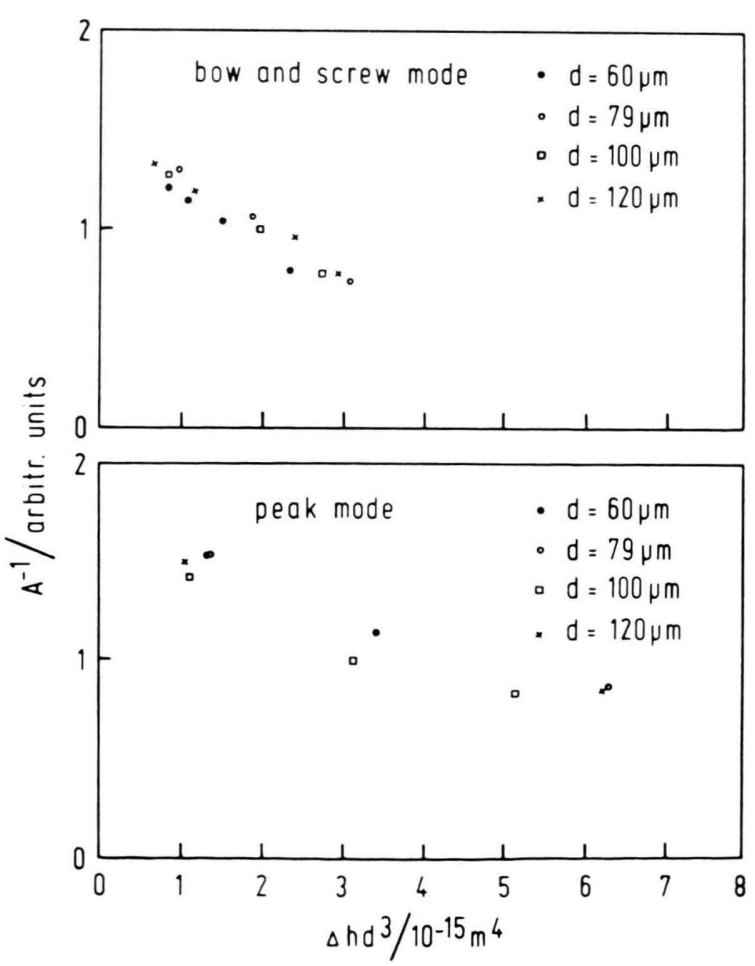

Fig. 11. Fitting parameter $A^{-1}$, proportional to the average viscosity $\langle\eta\rangle$, as a function of $\Delta h d^{3}(\Delta h=\Delta p / Q$ MBBA $)$. 
mode curve can be extended a little into the screw mode range but only for a metastable bow mode.

The reciprocal adaption factors $A^{-1}$ which are proportional to $\langle\eta\rangle$ are plotted in Fig. 11 as a function of $\Delta p d^{3}$. One observes that with increasing flow alignment, which means increasing $\Delta p d^{3}$, the mean viscosity $\langle\eta\rangle$ decreases monotonically, as has been expected. The absolute determination of $\langle\eta\rangle$ has not yet been possible because our experimental setup did not allow to measure the very small flow rates which range between $10^{-6} \mathrm{~cm}^{3} \mathrm{~s}^{-1}$ and $10^{-4} \mathrm{~cm}^{3} \mathrm{~s}^{-1}$.
1 P. Pieranski and E. Guyon, Phys. Rev. A 9, 404 [1974]. E. Guyon and P. Pieranski, J. Phys. 3 b, 203 [1975].

2 J. Wahl and F. Fischer, Mol. Cryst. Liquid Cryst. 22, 359 [1973].

${ }^{3}$ F. Fischer, Z. Naturforsch. 31 a, 302 [1976].
4 C. Mauguin, Phys. Z. 12, 1011 [1911].

5 Th. Waltermann and F. Fischer, Z. Naturforsch. 30 a, 519 [1975].

6 Th. Waltermann, to be published. 